\title{
Estudo comparativo entre tratamento endovascular e cirurgia convencional na correção eletiva de aneurisma de aorta abdominal: revisão bibliográfica
}

\author{
Endovascular elective treatment of the abdominal aortic aneurysm \\ versus conventional open repair: a comparative study
}

\author{
Ana Carolina P. Simão ${ }^{1}$, Ana Carolina de Alencar Gonçalves ${ }^{1}$, Milena Miguita Paulino ${ }^{1}$, Renata \\ Barbosa de Oliveira ${ }^{1}$, Camila Aparecida Polli ${ }^{1}$, Ayrton Cássio Fratezi ${ }^{2}$
}

\begin{abstract}
Resumo
O tratamento eletivo do aneurisma de aorta abdominal é recomendado pela alta morbiletalidade decorrente da eventual ruptura. O objetivo deste trabalho foi comparar o reparo endovascular eletivo com a cirurgia aberta e avaliar as mortalidades hospitalar e perioperatória, em 1 ano, por todas as causas e relacionadas ao aneurisma, a permanência hospitalar, as complicações, as taxas de sobrevida, conversão e reintervenção, a durabilidade do enxerto, o custo-benefício e a relação desses dados com o treinamento da equipe médica responsável pelo tratamento. Realizou-se uma revisão da literatura sobre reparo endovascular versus cirurgia convencional. Foram observados vantagem na sobrevivência perioperatória e menor estresse pós-cirúrgico; no entanto, os benefícios iniciais são perdidos por complicações e reintervenções tardias. Trabalhos baseados nas primeiras gerações de endopróteses superestimam as taxas de mortalidade em curto prazo, complicações e reintervenções. A durabilidade do enxerto, a real vantagem na sobrevida e o custo-benefício são incertos, e outros estudos são necessários para o seguimento em longo prazo.
\end{abstract}

Palavras-chave: Tratamento endovascular, aneurisma de aorta abdominal, cirurgia, prótese endovascular.

\section{Introdução}

$\mathrm{O}$ aneurisma de aorta abdominal (AAA) é definido como um alargamento da aorta de pelo menos $50 \%$ do diâmetro arterial, sendo recomendado tratamento eletivo quando atinge pelo menos $5,5 \mathrm{~cm}$ devido ao alto risco de ruptura $^{1}$ e potencial letalidade ${ }^{2}$. Seu diagnóstico, em geral, é incidental ou se dá em programas de rastreamento ${ }^{2}$. Com $\mathrm{o}$ intuito de diminuir a morbidade de uma eventual ruptura e a sua letalidade, que varia de 65 a $85 \%^{3}$, o tratamento eletivo é recomendado quando o tamanho do AAA atinge

\begin{abstract}
The elective treatment of the abdominal aortic aneurysm is recommended due to the high morbidity and mortality of a possible rupture. The objective of this study was to compare the elective endovascular aneurysm repair with open repair and to analyze the in-hospital and perioperative mortality rate during 1 year related to all causes and to the aneurysm, as well as the postoperative length of hospital stay, complications, survival rates, conversion and reintervention, graft durability, cost-benefit ratio, and relation with the medical team's experience. A review of the scientific literature about endovascular versus open repair was carried out. We found a higher rate of perioperative survival and less postoperative stress; nevertheless, the initial benefits were lost due to late complications and reinterventions. First-generation endografts overestimated the early results of mortality rates, complications, and reinterventions. Endograft durability, real advantage of survival rates, and cost-benefits are uncertain and further long-term follow-up studies are necessary.
\end{abstract}

Keywords: Endovascular treatment, abdominal aortic aneurysm, surgery, endograft.

$5,5 \mathrm{~cm}$ de diâmetro ${ }^{1}$ ou quando ocorre expansão do diâmetro transverso maior do que $5 \mathrm{~mm}$ em $1 \mathrm{ano}^{4}$.

O AAA acomete $4 \%$ dos homens e $1 \%$ das mulheres, sendo mais frequente na faixa etária dos 60 aos 70 anos de idade $^{4}$. Em estudo realizado no Hospital das Clínicas de São Paulo/SP, Puech-Leão et al. encontraram uma prevalência de 2,32\% de aneurismas incidentais na amostra estudada $^{5}$. Um estudo realizado no Reino Unido evidenciou uma prevalência de $0,3 \%$ de AAA na população masculina

1. Acadêmica de Medicina (5a ano), Faculdade de Medicina de Jundiaí (FMJ), Jundiaí, SP.

2. Professor adjunto, Disciplina de Cirurgia Vascular, Departamento de Cirurgia Geral, FMJ, Jundiaí, SP.

Trabalho apresentado no VIII Congresso Médico Acadêmico, realizado na Faculdade de Medicina de Jundiaí em 2008.

Não foram declarados conflitos de interesse associados à publicação deste artigo.

Artigo submetido em 10.09.08, aceito em 11.08.09.

J Vasc Bras. 2009;8(4):335-342.

Copyright $($ C 2009 by Sociedade Brasileira de Angiologia e de Cirurgia Vascular 
entre 50 e 64 anos, de $2,5 \%$ entre os 65 e 79 anos e de $4,1 \%$ em maiores de 80 anos $^{6}$.

Por mais de 50 anos, os AAA foram eletivamente tratados com reparo cirúrgico aberto, uma cirurgia de grande porte realizada sob anestesia geral exigindo pelo menos 30 min de clampeamento da aorta ${ }^{7}$ e profunda hipotermia. Devido à alta mortalidade associada com ruptura, à morte intraoperatória, à morbidade e comorbidades dos pacientes, pesquisas foram realizadas na procura de alternativas para o procedimento aberto clássico, sendo Parodi \& Palmaz os primeiros a relatarem o uso de técnica endovascular em $1991^{1,7-9}$. Esse procedimento baseia-se em duas pequenas incisões que expõem a artéria femoral por onde é introduzida, através de cateteres e fio guia, uma prótese no segmento aneurismático da aorta ${ }^{7,10}$. Desde então, essa opção de tratamento obteve ampla aceitação por ser uma alternativa menos invasiva ${ }^{7,8,10}$ e com potencial para reduzir a morbidade perioperatória, a mortalidade e o tempo de recuperação, principalmente em pacientes com alto risco cirúrgico para cirurgia clássica ${ }^{11,12}$. Antes do advento da cirurgia endovascular, a mortalidade perioperatória era de cerca de $8 \%$, decrescendo para $4 \%$ com o referido advento ${ }^{10}$.

Diversos trabalhos têm sido realizados na tentativa de avaliar os benefícios e complicações do reparo endovascular de aneurisma (REVA) no médio e longo prazo em comparação com a cirurgia aberta.

O objetivo geral do presente trabalho foi comparar o reparo endovascular com a cirurgia convencional em pacientes com AAA. Os objetivos específicos foram avaliar mortalidade hospitalar, mortalidade perioperatória, mortalidade em 1 ano, mortalidade por todas as causas, mortalidade relacionada ao aneurisma, permanência hospitalar, complicações, taxa de sobrevida, taxa de conversão, taxa de reintervenção, durabilidade do enxerto, custo-benefício e relação dessas variáveis com o treinamento da equipe médica responsável pelo tratamento.

\section{Material e método}

Realizou-se um levantamento bibliográfico nas bases de dados eletrônicos SciELO, MEDLINE e PubMed, no período de 1995 a 2008, com os descritores "tratamento endovascular", "aneurisma de aorta abdominal (cirurgia)", "prótese endovascular" e seus correspondentes termos em língua inglesa. Foram selecionados 32 artigos e lidos na ín- tegra. Dentre eles, 14 foram selecionados para esta revisão com base em ano de publicação, qualidade do artigo e dimensão da amostra.

\section{Resultados}

\section{Descrição dos estudos}

Na Tabela 1, observam-se as características dos estudos utilizados nesta revisão.

\section{Mortalidade hospitalar}

A porcentagem de mortalidade hospitalar foi de $2,1 \mathrm{e}$ $6,2 \%$, respectivamente, no grupo REVA e no de cirurgia aberta, resultando, então, uma taxa dois terços menor para o primeiro grupo ${ }^{7}$.

\section{Permanência hospitalar}

Em todos os artigos que compararam as modalidades de tratamento, houve menor tempo de permanência hospitalar ${ }^{1,3,7,10,11,15}$ para o REVA, com tempo médio de internação de 8,9 dias ${ }^{13}$. Em relação à necessidade de UTI, observaram-se melhores resultados no REVA ${ }^{10}$, com índice de 4 versus $7 \%$ na cirurgia aberta. Quanto ao tempo de permanência na UTI, verificou-se 2,8 dias para o REVA versus 6,5 dias para a cirurgia convencional ${ }^{1,11}$. A duração média da internação dos pacientes que faleceram 30 dias após a alta hospitalar foi de $47 \mathrm{dias}^{13}$.

\section{Mortalidade perioperatória}

Nos estudos utilizados para esta revisão, a mortalidade perioperatória - ocorrida dentro de 30 dias do pós-operatório - foi menor no grupo REVA em comparação com a cirurgia convencional, apresentando valores entre 1,2 e $14,9 \%{ }^{1,3,7,10,11,13,15}$. O EVAR Trial 1 evidenciou mortalidade perioperatória de $1,7 \%$ no REVA e de $4,7 \%$ no método convencional ${ }^{7}$. Outro estudo demonstrou valores semelhantes, com taxa de $1,2 \%$ no REVA e de $4,6 \%$ na cirurgia aberta $^{10}$.

\section{Mortalidade em 1 ano}

Os dados do estudo de Bush et al. revelam taxa de mortalidade em 1 ano de $8,7 \%$ para REVA e de $12,1 \%$ para cirurgia aberta ${ }^{11}$.

\section{Mortalidade por todas as causas}

A mortalidade global - causas relacionadas ou não ao aneurisma -, no estudo de Brewster et al., foi de $28,2 \%$, sendo $11 \%$ atribuído ao aneurisma ou ao tratamento. A 
Tabela 1 - Características dos estudos selecionados

\begin{tabular}{|c|c|}
\hline Estudo & Koning et al. ${ }^{13}$ \\
\hline Método & Estudo de coorte retrospectivo na base de dados EUROSTAR com 5.612 pacientes submetidos ao REVA \\
\hline Participantes & Idade média: $71 ; 93 \%$ do sexo masculino; DMA 5,5 cm \\
\hline Intervenção & REVA \\
\hline Estudo & Brewster et al. $^{8}$ \\
\hline Método & Revisão retrospectiva, duração de 12 anos, 873 pacientes com REVA \\
\hline Participantes & $\begin{array}{l}\text { Idade }<65,8,4 \% \text {; idade }>80,81,5 \% \text {; } 81,4 \% \text { do sexo masculino; DMA } 56,8 \mathrm{~cm} \text {; DLP } 43,5 \% \text {; DM } 12,4 \% \text {; TBG } \\
65,1 \% \text {; HAS } 73,4 \% \text {; DPOC } 24,1 \% \text {; DVP } 10,1 \% \text {; DCV 57,1\%; IRC 7\% }\end{array}$ \\
\hline Intervenção & REVA eletivo \\
\hline Estudo & Bush et al. ${ }^{11}$ \\
\hline Método & $\begin{array}{l}\text { Estudo retrospectivo na base de dados National Surgical Quality Improvement Program of the Department of Vet- } \\
\text { erans Affairs, duração de } 3 \text { anos, } 1.904 \text { pacientes ( } 717 \text { REVA e } 1.187 \text { aberta) }\end{array}$ \\
\hline Participantes & $\begin{array}{l}\text { Idade <60, } 7 \text { (REVA) x 10,3\% (aberta); idade > 80, 14,6 x 11,6\%; sexo masculino, 99,6 x 99,1\%; ASA } 2 \text { ou } 3,70,2 \\
\text { x 69,6\%; DM, 14,4 x 13,2\%; TBG, 39,5 x 45,7\%; IAM, 53,1 x 53,9\%; HAS, 74,2 x 75,6\% }\end{array}$ \\
\hline Intervenção & REVA ou cirurgia aberta \\
\hline Estudo & Brooks et al. ${ }^{3}$ \\
\hline Método & $\begin{array}{l}\text { Estudo randomizado controlado: EVAR Trial 1, } 1.082 \text { pacientes, } 543 \text { REVA x } 539 \text { cirurgia aberta; EVAR Trial } \\
\text { 2, } 338 \text { pacientes inaptos à cirurgia, } 166 \text { REVA x } 177 \text { observação }\end{array}$ \\
\hline Participantes & $\begin{array}{l}\text { EVAR Trial 1: idade média, 74; } 91 \% \text { do sexo masculino; DMA } 6,5 \mathrm{~cm} \text {; DM, } 9 \text { REVA x 12\% aberta; TBG, } 21 \text { x } \\
\text { 22\%; DCV, } 44 \text { x } 43 \% \\
\text { EVAR Trial 2: idade média, } 76 ; 85 \% \text { do sexo masculino; DMA } 6,5 \mathrm{~cm} \text {; DM, } 15 \text { REVA x 13\% observação; TBG, } 17 \\
\text { x 16\%; DCV, } 65 \text { x 73\% }\end{array}$ \\
\hline Intervenção & Cirurgia aberta ou REVA \\
\hline Estudo & Aarts et al. ${ }^{1}$ \\
\hline Método & $\begin{array}{l}\text { Estudo retrospectivo, pacientes submetidos à correção de AAA no Hospital Rijnstate (Holanda), duração de } 4 \\
\text { anos, } 215 \text { pacientes ( } 99 \text { REVA e } 116 \text { aberta) }\end{array}$ \\
\hline Participantes & $\begin{array}{l}\text { Idade } 70 \text { a 79: } 49 \text { (REVA) x 47\% (aberta); idade } 80 \text { a } 89: 23 \text { x 7\%; idade > 90: } 2 \text { x 1\%; sexo masculino, } 96 \text { x } 87 \% \text {; } \\
\text { DMA 5,77 x 6,01 cm; DM 7\%; DAC } 42 \text { x 29\%; HAS } 65 \text { x 61\% }\end{array}$ \\
\hline Intervenção & REVA ou cirurgia aberta \\
\hline Estudo & Blankensteijn et al. ${ }^{14}$ \\
\hline Método & Ensaio clínico randomizado multicêntrico, duração de 3 anos, 351 pacientes (173 REVA e 178 aberta) \\
\hline Participantes & $\begin{array}{l}\text { Idade média 70, } 7 \text { (REVA) x 69,6 (aberta); sexo masculino, 93,1 x 90,4\%; DM, 10,4 x 9,6\%; TBG, 64,2 x 55,1\%; } \\
\text { DCV, } 41 \text { x 46,6\%; HAS, 58,4 x 54,5\%; ASA 2, 70,5 x 61,8\% }\end{array}$ \\
\hline Intervenção & REVA ou cirurgia aberta \\
\hline Estudo & Michaels et al. $^{2}$ \\
\hline Método & Estudo randomizado: grupo 1, REVA x aberta; grupo 2, REVA x observação clínica \\
\hline Participantes & - \\
\hline Intervenção & REVA ou cirurgia aberta \\
\hline Estudo & Carvalho et al. ${ }^{4}$ \\
\hline Método & Estudo prospectivo, duração de 6 anos, 130 pacientes sem especificação da técnica cirúrgica \\
\hline Participantes & $\begin{array}{l}\text { Idade média: } 65 ; 88,5 \% \text { do sexo masculino; HAS } 63,8 \% \text {; TBG 53,8\%; DLP 52,3\%; DAC 31,5\%; DPOC 26,2\%; IRC } \\
\text { 10\%; DM 10\%; DCV 5,4\% }\end{array}$ \\
\hline Intervenção & Cirurgia eletiva \\
\hline
\end{tabular}


Tabela 1 (cont.) - Características dos estudos selecionados

\begin{tabular}{|c|c|}
\hline Estudo & Morris et al. ${ }^{6}$ \\
\hline Método & Ensaio clínico randomizado, duração de 4 anos, 1.082 pacientes (543 REVA e 539 aberta) \\
\hline Participantes & $\begin{array}{l}\text { Idade média: 74; } 91 \% \text { sexo masculino; DMA } 6,5 \mathrm{~cm} \text {; DM, } 9 \text { (REVA) x 12\% (aberta); TBG, } 21 \text { x 22\%; DCV, } 44 \text { x } \\
43 \%\end{array}$ \\
\hline Intervenção & REVA ou cirurgia aberta \\
\hline Estudo & Puech-Leão et al. ${ }^{5}$ \\
\hline Método & Estudo descritivo prospectivo, 2.756 voluntários submetidos a exame físico e USG abdominal \\
\hline Participantes & Idade $>50$, sem investigação prévia para AAA \\
\hline Intervenção & - \\
\hline Estudo & Prinssen et al. ${ }^{10}$ \\
\hline Método & Ensaio clínico randomizado, 345 pacientes (174 aberta e 171 REVA) \\
\hline Participantes & $\begin{array}{l}\text { Idade média, } 69,5 \text { (aberto) x 70,7 (REVA); sexo masculino, 90,2 (aberto) x 93\% (REVA); DMA, } 60 \text { (aberto) x } \\
60 \text { mm (REVA); DM, 9,8 (aberto) x 9,9\% (REVA); HAS, } 54 \text { (aberto) x 57,9\% (REVA); DCV, 46,6 (aberto) x } \\
40,9 \% \text { (REVA); TBG, } 54 \text { (aberto) x 64,9\% (REVA) }\end{array}$ \\
\hline Intervenção & REVA ou cirurgia aberta \\
\hline Estudo & Lee et al. ${ }^{15}$ \\
\hline Método & Revisão retrospectiva, 7.172 pacientes (4.607 aberta e 2.565 REVA) \\
\hline Participantes & $\begin{array}{l}\text { Idade média, } 71,9 \text { (aberta) e 73,4 (REVA); sexo masculino, 80,4\%; HAS, } 53 \text { (aberta) x 57\% (REVA); DM, } 11 \\
\text { (aberta e REVA); IRC, } 7 \text { (aberta) x 3\% (REVA); DPOC, } 29 \text { (aberta) x 25\% (REVA); DAC, } 14 \text { (aberta) x 20\% } \\
\text { (REVA); DVP, } 13 \text { (aberta) x 11\% (REVA) }\end{array}$ \\
\hline Intervenção & REVA ou cirurgia aberta \\
\hline
\end{tabular}

ASA = American Society of Anesthesiology; DAC = doença arterial coronariana; DCV = doença cardiovascular; DLP = dislipidemia; $\mathrm{DM}=$ diabetes melito; DMA = diâmetro médio do aneurisma; $\mathrm{DPOC}=$ doença pulmonar obstrutiva crônica; $\mathrm{DVP}=$ doença vascular periférica; HAS = hipertensão arterial sistêmica; IAM = infarto agudo do miocárdio; IRC = insuficiência renal crônica; REVA = reparo endovascular de aneurisma; TBG = tabagismo; USG = ultrassonografia.

maioria das mortes foi causada por doença cardíaca ou câncer ${ }^{8}$. Para Koning et al., a mortalidade total foi de $13 \%$, sendo que $2,5 \%$ dos óbitos estavam relacionados ao procedimento e $10,5 \%$ a outras causas ${ }^{13}$. Em Blankensteijn et al., observou-se maior mortalidade relacionada a causas cardiovasculares no grupo REVA em relação à cirurgia convencional, embora a diferença não seja estatisticamente significativa ${ }^{14}$. Em pacientes com grandes aneurismas, o tratamento com REVA alcançou uma redução estatisticamente significativa de dois terços na mortalidade após 30 dias da operação, quando comparado com a cirurgia aber$\mathrm{ta}^{3}$. A mortalidade global em 4 anos foi de $28 \%$ para ambos os grupos no EVAR Trial $1^{3}$.

\section{Mortalidade relacionada ao aneurisma}

A taxa cumulativa de mortalidade relacionada ao aneurisma - morte resultante de ruptura, infecção do enxerto, trombose ou reintervenção dentro ou após 30 dias do pro- cedimento original - foi de 5,7\% para a cirurgia clássica e de $2,1 \%$ para o REVA ${ }^{14}$. No EVAR Trial 2, não houve diferença na mortalidade relacionada ao aneurisma e a outras causas, pois ocorreram 23 mortes por ruptura de aneurisma no grupo de acompanhamento clínico e 13 mortes pósoperatórias e nove tardias por ruptura de aneurisma no grupo REVA ${ }^{3}$. Conforme Koning et al., a mortalidade relacionada ao procedimento foi de $1,6 \%$ por ano ${ }^{13}$.

\section{Complicações}

Os estudos de Bush et al., Aarts et al. e Prinssen et al. evidenciaram menor índice de complicação no grupo $\mathrm{REVA}^{1,10-12}$. A maior diferença relacionou-se à maior taxa de complicações pulmonares na cirurgia aberta ${ }^{10}$. Entretanto, a pesquisa EVAR Trial 1 encontrou maior taxa de complicações no REVA quando comparado com a cirurgia aberta, com 41 versus $9 \%$, respectivamente ${ }^{3}$. Lee sugere que a maior taxa de complicações pós-operatórias na cirur- 
gia aberta é contrabalanceada pelo aumento de complicações locais ou vasculares no REVA ${ }^{15}$.

As principais complicações das próteses disponíveis no mercado relacionam-se a endoleak, migração e trombose que resultam em altas taxas de reintervenções e falham na prevenção de ruptura do aneurisma ${ }^{11}$. O risco de ruptura anual do aneurisma após o REVA foi de 1 a 1,5\%, estando mais propensa a ocorrer em pacientes que apresentam endoleak ${ }^{7,8}$. A taxa de endoleak, em torno de $24 \%$, tem associação significativa com o índice de reintervenção ${ }^{1}$. Aarts e Prinssen demonstraram que há diferença significativa na perda sanguínea intraoperatória ${ }^{1,10}(150 \mathrm{~mL}$ no REVA versus $1.300 \mathrm{~mL}$ na cirurgia aberta) ${ }^{1}$.

\section{Durabilidade do enxerto}

Segundo o EVAR Trial 1, a durabilidade do enxerto varia de 20 a 30 anos $^{7}$.

\section{Custo-beneficio}

O custo por paciente no EVAR Trial 1 foi de cerca de US\$ 23.500 para o REVA e de US\$ 17.500 para a cirurgia clássica, enquanto que o gasto por paciente em 4 anos, no EVAR Trial 2, foi de aproximadamente US\$24.000 para o REVA e de US\$ 8.800 para o grupo do acompanhamento clínico $^{3}$. Lee et al. relata um custo de US\$ 50.346 para o REVA e de US\$ 47.009 para a cirurgia aberta ${ }^{15}$.

Considerando-se as taxas de readmissão em 30 dias e em 1 ano, obtiveram-se maiores índices para o REVA, com 12,4 e $38,8 \%$, respectivamente, contra 9,4 e $28,1 \%$ na cirurgia convencional ${ }^{11}$.

\section{Taxas de reintervenção}

Reintervenções podem ser necessárias em pelo menos $25 \%$ dos pacientes com $\mathrm{REVA}^{7}$, relacionando-se a endole$a k$ primário persistente, endoleak tardio, migração, trombose, aumento progressivo do AAA e obstrução. Em 12 anos, $10 \%$ dos pacientes necessitaram de reintervenção, sendo que o tempo livre de reintervenção foi de $78 \%$ em $5 \operatorname{anos}^{8}$. Intervenções secundárias após REVA foram necessárias em quase um quinto dos pacientes ${ }^{8}$.

A taxa de reintervenções durante 30 dias foi de $9,8 \%$ no REVA e de $5,8 \%$ na cirurgia aberta ${ }^{7}$, e, nos primeiros 9 meses, manteve-se maior taxa de reintervenção para o REVA $^{14}$. Nos EVAR Trial 1 e 2, observou-se que 20 e $26 \%$ dos pacientes, respectivamente, necessitaram de pelo menos uma reintervenção em 4 anos, enquanto no grupo de cirurgia aberta do EVAR Trial $1^{3}$ essa porcentagem foi de apenas 6\%. Contudo, o estudo de Aarts não encontrou diferença estatisticamente significativa na reintervenção entre os dois grupos ${ }^{1}$.

\section{Taxa de sobrevida}

A taxa de sobrevida global em 2 anos foi de $89,6 \%$ para a cirurgia convencional e de $89,7 \%$ para o REVA. A sobrevida sem complicações moderadas ou graves mostrou-se semelhante em ambos os grupos em 2 anos de acompanhamento $^{14}$. Demonstrou-se que o REVA pode melhorar a sobrevida após 3 anos de 79 para $86 \%$, com claro benefício de sobrevivência em curto prazo ${ }^{7}$. A sobrevida em 5 anos foi de 52\% no REVA, mas o EVAR Trial 1 e Blankensteijn mostraram que as vantagens de sobrevivência desapareceram enquanto que a mortalidade relacionada ao aneurisma permaneceu significativamente baixa em $2 \operatorname{anos}^{8}$. A sobrevivência em 8 anos de pacientes que foram submetidos ao REVA é de $70 \%{ }^{13}$.

O REVA resultou em uma sobrevivência relacionada ao aneurisma de 3\% a mais em 4 anos em relação à cirurgia convencional $^{3}$. Já o tempo livre de mortalidade relacionada ao aneurisma foi de $96 \%$ em 5 anos, e o relativo a todas as causas foi de $52 \%$ em 5 anos após o REVA ${ }^{8}$.

\section{Equipe médica e volume hospitalar}

Há correlação direta entre o maior fluxo hospitalar e a obtenção de melhores resultados no seguimento de pacientes submetidos à cirurgia aberta ${ }^{11}$.

Pacientes operados em hospitais de baixo fluxo, independente do tipo de cirurgia, tiveram aumento no risco de mortalidade perioperatória e em um ano. Não houve diferença significativa nas taxas de complicações entre hospitais de alto e baixo fluxo ${ }^{11}$.

Nos estudos iniciais (RETA e EUROSTAR), relataram-se maiores taxas de mortalidade precoce em relação aos estudos EVAR Trial 1 e 2. Um estudo observacional demonstrou resultados aceitáveis quando o REVA é disseminado do centro de treinamento universitário para a prática comunitária ${ }^{3}$.

\section{Taxa de conversão}

$\mathrm{Na}$ literatura, relata-se uma taxa de conversão de cerca de $2 \%{ }^{1}$, com conversão aguda de $2,3 \%$ e tardia de $1,7 \%$, relacionada a ruptura, migração, infecção ou crescimento do 
$\mathrm{AAA}^{8}$. Prinssen refere conversão intraoperatória para cirurgia aberta em $1,7 \%$ dos pacientes ${ }^{10}$.

\section{Discussão}

Observa-se que há vantagem na sobrevivência perioperatória com REVA se comparada a da cirurgia aberta ${ }^{14}$, o que pode ser explicado pelo fato de o tratamento endovascular ser menos invasivo e, portanto, resultar em menor resposta hipermetabólica após estresse. Durante a cirurgia aberta, o clampeamento da aorta implica em mudanças significativas nos parâmetros cardiovasculares tais como débito cardíaco, pressão arterial média e resistência vascular sistêmica, o que pode causar instabilidade hemodinâmica nos pacientes que apresentam cardiopatia prévia ou alto risco para doença cardiovascular ${ }^{11}$. A convergência da taxa de sobrevivência global no segundo ano após o reparo do aneurisma pode estar relacionada ao fato de que os pacientes que sobreviveram ao estresse da cirurgia aberta são menos propensos a morrer nos primeiros meses de pós-operatório do que aqueles tratados pelo REVA ${ }^{14}$.

$\mathrm{Na}$ maioria dos estudos, a taxa de mortalidade perioperatória é semelhante, exceto para Koning, que constatou maior valor para esta variáve $1^{13}$ com a técnica endovascular, o que pode ter sido influenciado pela alta prevalência de comorbidades cardiovasculares associadas.

Como os grupos submetidos ao REVA apresentavam mais comorbidades, as taxas de mortalidade em curto prazo, complicações e reintervenções podem estar superestimadas quando comparadas às da cirurgia aberta ${ }^{14}$. Ademais, muitos trabalhos foram baseados nas primeiras gerações de endopróteses e, por isso, também podem apresentar dados maiores de mortalidade, falhas, durabilidade e complicações ${ }^{8,14}$. Esses fatores podem justificar a similaridade dos índices de mortalidade entre as duas técnicas.

Além disso, a taxa de mortalidade perioperatória nos pacientes de cirurgia convencional é maior em relação ao REVA; no entanto, a menor mortalidade encontrada para o grupo REVA está limitada ao primeiro ano de pós-operatório $^{14}$, e, por conseguinte, a sobrevida em longo prazo é maior no grupo de cirurgia convencional. Vale ressaltar que os estudos realizados até hoje não seguiram em longo prazo os pacientes tratados por REVA; portanto, ainda não há dados que sustentem a vantagem desse método em relação à cirurgia aberta com respeito à sobrevida dos pacientes. Além disso, a mortalidade do grupo submetido a
REVA pode estar subestimada pelo menor tempo de permanência e pela subnotificação de óbitos em ambiente extra-hospitalar ${ }^{15}$.

Nos pacientes que apresentavam condições de serem submetidos a ambas as técnicas e que foram tratados por REVA, os benefícios iniciais na mortalidade são perdidos devido ao grande número de complicações e reintervenções tardias ${ }^{2}$. Contudo, Prinssen refere que nesses pacientes o REVA é a melhor opção por apresentar menores taxas de mortalidade perioperatória e complicações ${ }^{10}$. Já nos pacientes sem condições de sofrer a cirurgia aberta, as complicações iniciais mascaram a redução na mortalidade em longo prazo. Portanto, os resultados iniciais podem não demonstrar os reais benefícios do procedimento endovascular, sendo sempre necessário acompanhar a evolução dos pacientes para ter resultados mais fidedignos ${ }^{2}$.

Há divergência na literatura em relação aos benefícios do REVA para pacientes de alto risco que não podem ser submetidos à cirurgia convencional. Estudo retrospectivo sugere que o REVA é uma boa opção para pacientes com condições clínicas precárias (ASA classe 3 ou 4) e, desta forma, inelegíveis para o procedimento cirúrgico ${ }^{8,13}$. Em pacientes aptos para cirurgia aberta que foram submetidos ao REVA, demonstrou-se pequeno benefício adicional a um alto custo, mas o custo-benefício resultou alto em pacientes inaptos para cirurgia convencional. Um estudo randomizado, entretanto, demonstrou que em pacientes que podem fazer a cirurgia aberta, a opção pelo REVA não foi um procedimento seguro e nem efetivo, não trazendo também benefícios na sobrevivência dos pacientes de alto risco em 2 anos $^{3}$.

A literatura sugere que os casos de conversão ocorreram principalmente nos primeiros anos de REVA e foram relacionados ao grosso calibre e pouca flexibilidade da prótese, à seleção inapropriada dos pacientes, além de à pouca experiência e julgamento pobre por parte do cirurgião. Entretanto, nos últimos 4 anos houve redução na taxa conversão pelo uso de próteses de novas gerações e pela adoção de critérios de seleção mais apropriados ${ }^{8}$.

Apesar das melhoras nas endopróteses, problemas como endoleak tardio, migração ou aumento do AAA podem ocorrer e estão claramente relacionados à falha do REVA em excluir o AAA e prevenir a ruptura ${ }^{8,14}$. Todavia, a história natural e o comportamento do AAA indicam que, quando não tratado, há risco de ruptura de $11 \%$ ao ano 
e taxa de crescimento anual de $10 \%$, em oposição ao risco de ruptura de $1 \%$ após o REVA ${ }^{8}$.

Os trabalhos demonstram um alto índice de reintervenções em relação à cirurgia aberta, mas esses procedimentos secundários, quando realizados com sucesso, não comprometem a mortalidade relacionada ao aneurisma ou o seguimento tardio ${ }^{8}$. A necessidade de reintervenções somada ao alto valor da prótese encarece o REVA ${ }^{3}$, tornando-o mais caro que a cirurgia clássica, apesar de esta requerer maior tempo de internação ${ }^{1,15}$.

Em estudo retrospectivo não randomizado, o REVA mostrou diversas vantagens sobre o reparo cirúrgico aberto em curto prazo, porém ainda há dúvidas quanto à durabilidade dos enxertos ${ }^{13}$, mesmo com o desenvolvimento tecnológico. Além disso, a mortalidade associada ao reparo do aneurisma pode ser reduzida com o aperfeiçoamento decorrente da maior difusão deste método ${ }^{10}$.

Os benefícios encontrados no REVA incluem menor perda sanguínea e necessidade de transfusão, tempos de procedimento, de internação em UTI e em enfermaria mais curtos, menor taxa de eventos adversos no pós-operatório imediato e recuperação mais rápida ${ }^{8}$. Além disso, pacientes submetidos ao REVA tiveram melhor seguimento estatisticamente significativo, apresentando menores taxas de mortalidade em curto prazo ${ }^{11}$. No entanto, esses benefícios podem não ser sustentados, já que reintervenções podem ser necessárias em pelo menos $25 \%$ dos pacientes submetidos ao REVA? ${ }^{7}$.

Pelo exposto acima, o REVA é um procedimento que demanda programas de treinamento ainda não disponíveis em muitos centros de cirurgia vascular. Com a seleção adequada do paciente e a utilização de próteses de geração mais recente, o REVA pode alcançar com sucesso as metas do reparo de AAA com mortalidades perioperatória e em longo prazo satisfatórias e menor morbidade, apesar de ser utilizado em uma população de alto risco relativo.

É de fundamental importância o treinamento e aperfeiçoamento da equipe médica, além da detalhada análise dos pacientes, não se devendo subestimar a sua preferência após uma clara explicação acerca da técnica, as suas vantagens e desvantagens.

\section{Conclusão}

Há benefícios em curto prazo do REVA quando comparado à cirurgia convencional, porém a vantagem é perdida após o primeiro ano.

O REVA apresenta menores taxas de mortalidade hospitalar, permanência hospitalar, mortalidade por todas as causas, mortalidade relacionada ao aneurisma, mortalidade perioperatória e mortalidade em 1 ano. Entretanto, apresenta maiores índices de complicações e reintervenção. A durabilidade do enxerto, real vantagem na sobrevida e no custo-benefício são incertos, e outros estudos são necessários para o seguimento em longo prazo. Treinamento e aperfeiçoamento da equipe médica influem nos resultados após o REVA.

\section{Referências}

1. Aarts F, Sterkenburg SV, Blankensteijn JD. Endovascular aneurysm repair versus open aneurysm repair: comparison of treatment outcome and procedure-related reintervention rate. Ann Vasc Surg. 2005;19:699-704.

2. Michaels JA, Drury D, Thomas SM. Cost-effectiveness of endovascular abdominal aortic aneurysm repair. Br J Surg. 2005;92:960-7.

3. Brooks MJ, Brown LC, Greenhalgh RM. Defining the role of endovascular therapy in the treatment of abdominal aortic aneurysm: results of a prospective randomized trial. Adv Surg. 2006;40:191-204.

4. Carvalho FC, Brito VP, Tribulatto EC, van Bellen B. Estudo prospectivo da morbi-mortalidade precoce e tardia da cirurgia do aneurisma da aorta abdominal. Arq Bras Cardiol. 2005;84:292-6.

5. Puech-Leão P, Molnar LJ, Oliveira IR, Cerri GG. Prevalence of abdominal aortic aneurysms: a screening program in São Paulo, Brazil. Sao Paulo Med J. 2004;122:158-60.

6. Morris GE, Hubbard CS, Quick CR. An abdominal aortic aneurysm screening programme for all males over the age of 50 years. Eur J Vasc Surg. 1994;8:156-60.

7. Greenhalgh RM, Brown LC, Kwong GP, Powell JT, Thompson SG, EVAR Trial Participants. Comparison of endovascular aneurysm repair with open repair in patients with abdominal aortic aneurysm (EVAR trial 1), 30-day operative mortality results: randomised controlled trial. Lancet. 2004;364:843-8.

8. Brewster DC, Jones JE, Chung TK, et al. Long-term outcomes after endovascular abdominal aortic aneurysm repair: the first decade. Ann Surg. 2006;244:426-38.

9. Parodi JC, Palmaz IC. Transfemural intraluminal graft implantation for abdominal aortic aneurysms. Ann Vasc Surg. 1991;5:491-9.

10. Prinssen M, Verhoeven EL, Buth J, et al. A randomized trial comparing conventional and endovascular repair of abdominal aortic aneurysms. N Engl J Med. 2004;351:1607-18.

11. Bush RL, Johnson ML, Collins TC, et al. Open versus endovascular abdominal aortic aneurysm repair in VA hospitals. J Am Coll Surg. 2006;202:577-87. 
12. Alves CM, Souza JA. Estado da arte em stents de aorta. Rev Soc Cardiol Estado Sao Paulo. 2005; 15:362-8.

13. Koning GG, Vallabhaneni SR, van Marrewijk CJ, Leurs LJ, Laheij RJ, Buth J. Mortalidade relacionada ao tratamento endovascular do aneurisma da aorta abdominal com o uso dos modelos revisados. Rev Bras Cir Cardiovasc. 2007;22:7-14.

14. Blankensteijn JD, de Jong SE, Prinssen M, et al. Two-year outcomes after conventional or endovascular repair of abdominal aortic aneurysms. N Engl J Med. 2005;352:2398-405.

15. Lee WA, Carter JW, Upchurch G, Seeger JM, Huber TS. Perioperative outcomes after open and endovascular repair of intact abdominal aortic aneurysms in the United States during 2001. J Vasc Surg. 2004;39:491-6.

Correspondência:

Ana Carolina Pasquini Simão

Atalaia Velha, 298, Alto da Mooca

CEP 03191-140 - São Paulo, SP

Tel.: (11) 2965.5308, (11) 8695.2194

E-mail: anacarol_pasquini@yahoo.com.br

\section{Contribuições dos autores}

Concepção e desenho do estudo: ACF

Análise e interpretação dos dados: ACPS, ACAG

Coleta de dados: ACPS, ACAG, CAP, MMP, RBO

Redação do artigo: ACPS, ACAG, CAP, MMP, RBO

Revisão crítica do texto: ACF

Aprovação final do artigo*: ACPS, ACAG, CAP, MMP, $\mathrm{RBO}, \mathrm{ACF}$

Análise estatística: ACPS, ACAG, CAP, MMP, RBO

Responsabilidade geral pelo estudo: $\mathrm{ACF}$

Informações sobre financiamento: $\mathrm{ACF}$

* Todos os autores leram e aprovaram a versão final submetida ao J Vasc Bras. 